Article

\title{
Stolarsky Means in Many Variables
}

\section{Slavko Simić 1,2,* and Bandar Bin-Mohsin ${ }^{3}$}

1 Nonlinear Analysis Research Group, Ton Duc Thang University, Ho Chi Minh City 758307, Vietnam

2 Faculty of Mathematics and Statistics, Ton Duc Thang University, Ho Chi Minh City 758307, Vietnam

3 Department of Mathematics, College of Science, King Saud University, Riyadh 11451, Saudi Arabia; balmohsen@ksu.edu.sa

* Correspondence: slavkosimic@tdtu.edu.vn

Received: 14 July 2020; Accepted: 6 August 2020; Published: 8 August 2020

Abstract: We give in this article two possible explicit extensions of Stolarsky means to the multi-variable case. They attain all main properties of Stolarsky means and coincide with them in the case of two variables.

Keywords: extended mean values; logarithmic convexity; multi-variable case

MSC: 26A51; 60E15

\section{Introduction}

There is a huge number of papers investigating properties of the so-called Stolarsky (or extended) two-parametric mean values, defined for positive variables $x, y ; x \neq y$, as

$$
E_{r, s}(x, y):=\left(\frac{r\left(x^{s}-y^{s}\right)}{s\left(x^{r}-y^{r}\right)}\right)^{1 /(s-r)}, \quad r s(r-s) \neq 0 .
$$

Those means can be continuously extended on the domain

$$
\left\{(r, s ; x, y) \mid r, s \in \mathbb{R} ; x, y \in \mathbb{R}_{+}\right\}
$$

by the following

$$
E_{r, s}(x, y)= \begin{cases}\left(\frac{r\left(x^{s}-y^{s}\right)}{s\left(x^{r}-y^{r}\right)}\right)^{1 /(s-r)}, & r s(r-s) \neq 0 ; \\ \exp \left(-\frac{1}{s}+\frac{x^{s} \log x-y^{s} \log y}{x^{s}-y^{s}}\right), & r=s \neq 0 ; \\ \left(\frac{x^{s}-y^{s}}{s(\log x-\log y)}\right)^{1 / s}, & s \neq 0, r=0 ; \\ \sqrt{x y}, & r=s=0 ; \\ x, & y=x>0\end{cases}
$$

and in this form has been introduced by Keneth Stolarsky in [1].

Most of the classical two variable means are just special cases of the class E. For example, $E_{1,2}=\frac{x+y}{2}$ is the arithmetic mean, $E_{-r, r}=E_{0,0}=\sqrt{x} y$ is the geometric mean, $E_{0,1}=\frac{x-y}{\log x-\log y}$ is the logarithmic mean, $E_{1,1}=\left(x^{x} / y^{y}\right)^{\frac{1}{x-y}} / e$ is the identric mean, etc. More generally, the $r$-th power mean $\left(\frac{x^{r}+y^{r}}{2}\right)^{1 / r}$ is equal to $E_{r, 2 r}([2])$.

Characteristic properties of Stolarsky means are:

1. Symmetry in variables, $E_{r, s}(x, y)=E_{r, s}(y, x)$;

2. Symmetry in parameters, $E_{r, s}(x, y)=E_{s, r}(x, y)$; 
3. Means $E_{r, s}(x, y)$ are homogeneous of order one i.e, $E_{r, s}(t x, t y)=t E_{s, r}(x, y), t>0$.

4. Means $E_{r, s}(x, y)$ are monotone increasing in both parameters $r$ and $s$.

By two articles $([3,4])$ published in Amer.Math. Monthly, this class of means attains popularity in a wide audience. As a result, great number of papers are produced investigating its most subtle properties. In this sense we quote here papers [5,6]. A comparison of Stolarsky and Gini means is given in [7-9], weighted variants in [10,11]. F. Qi in [12] find intervals of $r, s$ where these means are logarithmically convex/concave, etc.

Furthermore, there are several papers attempting to define an extension of the class $E$ to $n, n>2$ variables. Unfortunately, this is done in a highly implicit mode $([5,6,13-15])$.

Here is an illustration of this point; J. Merikoski ([13]) has proposed the following generalization of the Stolarsky mean $E_{r, s}$ to several variables

$$
E_{r, s}(X):=\left[\frac{L\left(X^{s}\right)}{L\left(X^{r}\right)}\right]^{\frac{1}{s-r}}, r \neq s,
$$

where $X=\left(x_{1}, \cdots, x_{n}\right)$ is an $n$-tuple of positive numbers and

$$
L\left(X^{s}\right):=(n-1) ! \int_{I_{n-1}} \prod_{i=1}^{n} x_{i}^{s u_{i}} d u_{1} \cdots d u_{n-1} .
$$

The symbol $I_{n-1}$ stands for the Euclidean simplex which is defined by

$$
I_{n-1}:=\left\{\left(u_{1}, \cdots, u_{n-1}\right): u_{i} \geq 0,1 \leq i \leq n-1 ; u_{1}+\cdots+u_{n-1} \leq 1\right\} .
$$

In this article we shall expose two possible explicit formulae of Stolarsky means in $n \geq 2$ variables which preserve its main properties and coincide for $n=2$.

The first one is given by the following

Let $X_{n}=\left(x_{1}, x_{2}, \ldots, x_{n}\right) \in \mathbb{R}_{+}^{n}$. Then,

$$
e_{r, s}\left(X_{n}\right)=e_{r, s}\left(x_{1}, x_{2}, \ldots, x_{n}\right):=\left(\frac{r^{2}}{s^{2}} \frac{x_{1}^{n s}+x_{2}^{n s}+\ldots+x_{n}^{n s}-n\left(x_{1} x_{2} \ldots x_{n}\right)^{s}}{x_{1}^{n r}+x_{2}^{n r}+\ldots+x_{n}^{n r}-n\left(x_{1} x_{2} \ldots x_{n}\right)^{r}}\right)^{\frac{1}{n(s-r)}}, r s(s-r) \neq 0
$$

represents an extension of Stolarsky means to the multi-variable case.

Remark 1. We assume that there exist $j, k ; 1 \leq j<k \leq n$, such that $x_{j} \neq x_{k}$.

It is of interest to examine the inner structure of those means. For example, applying the formula

$$
x^{3}+y^{3}+z^{3}-3 x y z=\frac{1}{2}(x+y+z)\left[(x-y)^{2}+(y-z)^{2}+(z-x)^{2}\right],
$$

we obtain that

$$
e_{r, s}\left(x_{1}, x_{2}, x_{3}\right)=\left(A_{r, s}\left(x_{1}, x_{2}, x_{3}\right)\right)^{1 / 3}\left(B_{r, s}\left(x_{1}, x_{2}, x_{3}\right)\right)^{2 / 3}
$$

where

$$
A_{r, s}\left(x_{1}, x_{2}, x_{3}\right):=\left(\frac{x_{1}^{s}+x_{2}^{s}+x_{3}^{s}}{x_{1}^{r}+x_{2}^{r}+x_{3}^{r}}\right)^{1 /(s-r)}
$$

is the well-known Gini mean, and

$$
B_{r, s}\left(x_{1}, x_{2}, x_{3}\right):=\left(\frac{r^{2}}{s^{2}} \frac{\left(x_{1}^{s}-x_{2}^{s}\right)^{2}+\left(x_{2}^{\mathcal{S}}-x_{3}^{\mathcal{S}}\right)^{2}+\left(x_{3}^{\mathcal{s}}-x_{1}^{\mathcal{s}}\right)^{2}}{\left(x_{1}^{r}-x_{2}^{r}\right)^{2}+\left(x_{2}^{r}-x_{3}^{r}\right)^{2}+\left(x_{3}^{r}-x_{1}^{r}\right)^{2}}\right)^{1 /(2(s-r))}
$$


is the new mean in 3 variables which coincides with the Stolarsky mean $E_{r, s}\left(x_{1}, x_{2}\right)$ whenever $x_{3}=x_{1}$ or $x_{3}=x_{2}$.

This notion leads to the second, more general representation of Stolarsky means in many variables.

Let $A_{n}=\left(a_{1}, a_{2}, \ldots, a_{n}\right), X_{n}=\left(x_{1}, x_{2}, \ldots, x_{n}\right), Y_{n}=\left(y_{1}, y_{2}, \ldots, y_{n}\right) ; A_{n}, X_{n}, Y_{n} \in \mathbb{R}_{+}^{n}$.

Then

$$
E_{r, s}\left(A_{n} ; X_{n}, Y_{n}\right):=\left(\frac{r^{2}}{s^{2}} \frac{a_{1}\left(x_{1}^{\mathcal{S}}-y_{1}^{s}\right)^{2}+a_{2}\left(x_{2}^{\mathcal{s}}-y_{2}^{s}\right)^{2}+\cdots+a_{n}\left(x_{n}^{\mathcal{S}}-y_{n}^{s}\right)^{2}}{a_{1}\left(x_{1}^{r}-y_{1}^{r}\right)^{2}+a_{2}\left(x_{2}^{r}-y_{2}^{r}\right)^{2}+\cdots+a_{n}\left(x_{n}^{r}-y_{n}^{r}\right)^{2}}\right)^{\frac{1}{2(s-r)}}
$$

represents another multi-variable variant of Stolarsky means.

It will be shown in the sequel that both means $e_{r, s}\left(X_{n}\right)$ and $E_{r, s}\left(A_{n} ; X_{n}, Y_{n}\right)$ are monotone increasing in parameters $r$ and $s$. An intriguing task is to determine some necessary and sufficient conditions for their monotonicity in $n$. Although the solution is relatively simple in the second case and reduces to the monotonicity of sequences $X_{n}$ and $Y_{n}$ (independently of $A_{n}$ ), this question is much more complicated for the means $e_{r, s}\left(X_{n}\right)$.

For example, means $e_{0,0}\left(X_{n}\right)$ are monotone increasing/decreasing in $n$ if and only if $x_{n} \gtrless g\left(z\left(X_{n-1}\right), e_{0,0}\left(X_{n-1}\right)\right), n \geq 3$, where $z\left(X_{n}\right)$ is the geometric mean of numbers $X_{n}$ and $g\left(z_{n}, e_{0,0}\left(X_{n}\right)\right):=z_{n}\left(e_{0,0}\left(X_{n}\right) / z_{n}\right)^{3(n+1) /(n+2)}$.

\section{Results and Proofs}

Recall that the Jensen functional $J_{n}(p, x ; f)$ is defined on an interval $I \subseteq \mathbb{R}$ by

$$
J_{n}(p, x ; f):=\sum_{1}^{n} p_{i} f\left(x_{i}\right)-f\left(\sum_{1}^{n} p_{i} x_{i}\right)
$$

where $f: I \rightarrow \mathbb{R}, x=\left(x_{1}, x_{2}, \cdots, x_{n}\right) \in I^{n}$ and $p=\left\{p_{i}\right\}_{1}^{n}$ is a positive weight sequence.

Another well known assertion is the following

Jensen's inequality: If $f$ is twice continuously differentiable and $f^{\prime \prime} \geq 0$ on an interval $I$, then $f$ is convex on I and the inequality

$$
J_{n}(p, x ; f)=\sum_{1}^{n} p_{i} f\left(x_{i}\right)-f\left(\sum_{1}^{n} p_{i} x_{i}\right) \geq 0
$$

holds for each $x:=\left(x_{1}, \ldots, x_{n}\right) \in I^{n}$ and any positive weight sequence $p:=\left\{p_{i}\right\}_{1}^{n}$ with $\sum_{1}^{n} p_{i}=1$.

The next two properties of Jensen functionals will be of importance in the sequel.

Theorem 1 ([16,17]). Let $f, g: I \rightarrow \mathbb{R}$ be twice continuously differentiable functions. Assume that $g$ is strictly convex and $\phi$ is a continuous and strictly monotone function on $I$.

Then the expression

$$
\phi^{-1}\left(\frac{J_{n}(p, x ; f)}{J_{n}(p, x ; g)}\right),(n \geq 2),
$$

represents a mean value of the numbers $x_{1}, \cdots, x_{n} \in I$, that is

$$
\min \left\{x_{1}, \cdots, x_{n}\right\} \leq \phi^{-1}\left(\frac{J_{n}(p, x ; f)}{J_{n}(p, x ; g)}\right) \leq \max \left\{x_{1}, \cdots, x_{n}\right\},
$$

if and only if the relation

$$
f^{\prime \prime}(t)=\phi(t) g^{\prime \prime}(t)
$$

holds for each $t \in I$. 
Theorem 2 ([18]). Let $f_{s}$ be a twice continuously differentiable function on the interval $J:=(c, d)$ for each parameter $s \in I:=(a, b)$. If $s \rightarrow f_{s}^{\prime \prime}(x)$ is log-convex on I for each $x \in J$, then the expression

$$
s \rightarrow \Phi_{f}(w, x ; s):=\sum_{1}^{n} w_{i} f_{s}\left(x_{i}\right)-f_{s}\left(\sum_{1}^{n} w_{i} x_{i}\right),
$$

is $\log$-convex on I for each $x:=\left(x_{1}, \ldots, x_{n}\right) \in J^{n}$, where $w=\left\{w_{i}\right\}_{1}^{n}$ is any positive weight sequence.

Lemma 1. A function $F$ is convex on an interval I if and only if the ratio

$$
\frac{F(s)-F(r)}{s-r}
$$

is monotone increasing in both $r$ and $s$ for $r, s \in I$.

In the following two theorems we shall prove that our expressions $e_{r, s}\left(X_{n}\right)$ and $E_{r, s}\left(A_{n} ; X_{n}, Y_{n}\right)$, extended to the whole $(r, s)$ plane, are actually means which preserve all main properties of the ordinary Stolarsky means and coincide with them for $n=2$.

Theorem 3. Let,

$$
e_{r, s}\left(x_{1}, x_{2}, \ldots, x_{n}\right)= \begin{cases}\left(\frac{r^{2}\left(\sum_{1}^{n} x_{i}^{n s}-n\left(\prod_{1}^{n} x_{i}\right)^{s}\right)}{s^{2}\left(\sum_{1}^{n} x_{i}^{n r}-n\left(\prod_{1}^{n} x_{i}\right)^{r}\right)}\right)^{1 /(n(s-r))} & , r s(s-r) \neq 0 ; \\ \left(\frac{2}{n s^{2}} \frac{\sum_{1}^{n} x_{i}^{n s}-n\left(\prod_{1}^{n} x_{i}\right)^{s}}{n \sum_{1}^{n} \log ^{2} x_{i}-\left(\sum_{1}^{n} \log x_{i}\right)^{2}}\right)^{1 /(n s)} & , r=0, s \neq 0 \\ \exp \left(\frac{-2}{n s}+\frac{\sum_{1}^{n} x_{i}^{n s} \log x_{i}\left(\sum_{1}^{n} \log x_{i}\right)\left(\prod_{1}^{n} x_{i}\right)^{s}}{\sum_{1}^{n} x_{i}^{n s}-n\left(\prod_{1}^{n} x_{i}\right)^{s}}\right) & , r=s \neq 0 \\ \exp \left(\frac{n^{2} \sum_{1}^{n} \log ^{3} x_{i}-\left(\sum_{1}^{n} \log x_{i}\right)^{3}}{3 n\left(n \sum_{1}^{n} \log ^{2} x_{i}-\left(\sum_{1}^{n} \log x_{i}\right)^{2}\right)}\right) & , r=s=0 .\end{cases}
$$

Then

1. Expressions $e_{r, s}\left(X_{n}\right)$ are means, that is,

$$
\min \left\{x_{1}, x_{2}, \ldots, x_{n}\right\} \leq e_{r, s}\left(x_{1}, x_{2}, \ldots, x_{n}\right) \leq \max \left\{x_{1}, x_{2}, \ldots, x_{n}\right\} .
$$

2. $e_{r, s}\left(X_{n}\right)$ are symmetric in parameters $r$ and s i.e., $e_{r, s}\left(X_{n}\right)=e_{s, r}\left(X_{n}\right)$.

3. $e_{r, s}\left(X_{n}\right)$ are symmetric in all variables.

4. $e_{r, s}\left(X_{n}\right)$ are homogeneous of order one.

5. $e_{r, s}\left(X_{n}\right)$ are monotone increasing in both parameters $r$ and $s$.

6. $e_{r, s}\left(x_{1}, x_{2}\right)=E_{r, s}\left(x_{1}, x_{2}\right)$.

Proof. Note that the Properties $2-4$ are evident and can be proved directly.

We apply Theorem A for the proof of Property 1.

Namely, choose that $g=f_{r}(y)$ and

$$
f=f_{s}(y):= \begin{cases}\left(e^{s y}-s y-1\right) / s^{2} & , s \neq 0 ; \\ y^{2} / 2 & , s=0 .\end{cases}
$$

The conditions of Theorem A are fulfilled with

$$
f^{\prime \prime}(y)=e^{s y}, g^{\prime \prime}(y)=e^{r y}, \phi(y)=e^{(s-r) y}, \phi^{-1}(y)=\frac{1}{s-r} \log y,
$$

for $r \neq s$.

Therefore, with $p_{i}=1 / n$, we obtain 


$$
\min \left\{y_{i}\right\}_{1}^{n} \leq \frac{1}{s-r} \log \left(\frac{r^{2}}{s^{2}} \frac{\sum_{1}^{n} e^{s y_{i}}-n e^{\left(\sum_{1}^{n} y_{i}\right) s / n}}{\sum_{1}^{n} e^{r y_{i}}-n e^{\left(\sum_{1}^{n} y_{i}\right) r / n}}\right) \leq \max \left\{y_{i}\right\}
$$

that is,

$$
e^{\min \left\{y_{i}\right\}_{1}^{n}} \leq\left(\frac{r^{2}}{s^{2}} \frac{\sum_{1}^{n} e^{s y_{i}}-n e^{\left(\sum_{1}^{n} y_{i}\right) s / n}}{\sum_{1}^{n} e^{r y_{i}}-n e^{\left(\sum_{1}^{n} y_{i}\right) r / n}}\right)^{1 /(s-r)} \leq e^{\max \left\{y_{i}\right\}_{1}^{n}} .
$$

In the case $r=0, s \neq 0$, we have

$$
f^{\prime \prime}(y)=e^{s y}, g^{\prime \prime}(y)=1, \phi(y)=e^{s y}, \phi^{-1}(y)=\frac{1}{s} \log y .
$$

Hence,

$$
e^{\min \left\{y_{i}\right\}_{1}^{n}} \leq\left(\frac{2 n}{s^{2}} \frac{\sum_{1}^{n} e^{s y_{i}}-n e^{\left(\sum_{1}^{n} y_{i}\right) s / n}}{n \sum_{1}^{n} y_{i}^{2}-\left(\sum_{1}^{n} y_{i}\right)^{2}}\right)^{1 / s} \leq e^{\max \left\{y_{i}\right\}_{1}^{n}} .
$$

Now, change of variables $e^{y_{i}}=x_{i}, s \rightarrow n s, r \rightarrow n r$, evidently leads to the desired results.

For the proof of Property 5. we shall use Theorem B.

By the function $f_{s}(y)$ defined above, we have that $f_{s}^{\prime \prime}(y)=e^{s y}$ is log-convex for $s \in \mathbb{R}$.

Hence, by Theorem B we obtain that the form

$$
F(s)=\frac{\sum_{1}^{n} e^{s y_{i}}-n e^{\left(\sum_{1}^{n} y_{i}\right) s / n}}{n s^{2}}
$$

is log-convex on $\mathbb{R}$.

Since a positive function is log-convex on $I$ if its logarithm is convex on $I$, applying Lemma 1 we have that the form

$$
\frac{\log F(s)-\log F(r)}{s-r}=\log \left(\frac{r^{2} \sum_{1}^{n} e^{s y_{i}}-n e^{\left(\sum_{1}^{n} y_{i}\right) s / n}}{\sum_{1}^{n} e^{r y_{i}}-n e^{\left(\sum_{1}^{n} y_{i}\right) r / n}}\right)^{1 /(s-r)},
$$

is monotone increasing in both $r$ and $s$.

The same change of variables $e^{y_{i}}=x_{i}, s \rightarrow n s, r \rightarrow n r$, proves the validity of Property 5 .

Finally, for the Property 6. of Theorem 3, we have

$$
\begin{gathered}
e_{r, s}\left(x_{1}, x_{2}\right)=\left(\frac{r^{2}}{s^{2}} \frac{x_{1}^{2 s}+x_{2}^{2 s}-2\left(x_{1} x_{2}\right)^{s}}{x_{1}^{2 r}+x_{2}^{2 r}-2\left(x_{1} x_{2}\right)^{r}}\right)^{1 /(2(s-r))} \\
=\left(\frac{r^{2}}{s^{2}} \frac{\left(x_{1}^{s}-x_{2}^{s}\right)^{2}}{\left(x_{1}^{r}-x_{2}^{r}\right)^{2}}\right)^{1 /(2(s-r))}=\left|\frac{r}{s} \frac{\left(x_{1}^{s}-x_{2}^{s}\right)}{\left(x_{1}^{r}-x_{2}^{r}\right)}\right|^{1 /(s-r)}=E_{r, s}\left(x_{1}, x_{2}\right) .
\end{gathered}
$$

Theorem 4. Let,

$$
E_{r, s}\left(A_{n} ; X_{n}, Y_{n}\right)= \begin{cases}\left(\frac{r^{2}\left(\sum_{1}^{n} a_{i}\left(x_{i}^{s}-y_{i}^{s}\right)^{2}\right.}{s^{2}\left(\sum_{1}^{n} a_{i}\left(x_{i}^{r}-y_{i}^{r}\right)^{2}\right.}\right)^{1 /(2(s-r))} & , r s(s-r) \neq 0 ; \\ \left(\frac{\sum_{1}^{n} a_{i}\left(x_{i}^{s}-y_{i}^{s}\right)^{2}}{s^{2} \sum_{1}^{n} a_{i}\left(\log x_{i}-\log y_{i}\right)^{2}}\right)^{1 /(2 s)} & , r=0, s \neq 0 ; \\ \exp \left(\frac{-1}{s}+\frac{\sum_{1}^{n} a_{i}\left(x_{i}^{s}-y_{i}^{s}\right)\left(x_{i}^{s} \log x_{i}-y_{i}^{s} \log y_{i}\right)}{\sum_{1}^{n} i_{i}\left(x_{i}^{s}-y_{i}^{s}\right)^{2}}\right) & , r=s \neq 0 ; \\ \exp \left(\frac{\sum_{1}^{n} a_{i}\left(\log x_{i}-\log y_{i}\right)\left(\log x_{i}-\log ^{2} y_{i}\right)}{2 \sum_{1}^{n} a_{i}\left(\log x_{i}-\log y_{i}\right)^{2}}\right) & , r=s=0 .\end{cases}
$$

Then

1. Functions $E_{r, s}\left(A_{n} ; X_{n}, Y_{n}\right)$ are means. 
2. Means $E_{r, s}\left(A_{n} ; X_{n}, Y_{n}\right)$ are symmetric in parameters $r$ and $s$.

3. Means $E_{r, s}\left(A_{n} ; X_{n}, Y_{n}\right)$ are symmetric in variables, that is, $E_{r, s}^{n}\left(A_{n} ; X_{n}, Y_{n}\right)=E_{r, s}^{n}\left(A_{n} ; Y_{n}, X_{n}\right)$.

4. Means $E_{r, s}\left(A_{n} ; X_{n}, Y_{n}\right)$ are homogeneous of order one.

5. $E_{r, s}\left(A_{n} ; X_{n}, Y_{n}\right)$ are monotone increasing in both parameters $r$ and $s$.

6. $E_{r, s}\left(a_{1} ; x_{1}, y_{1}\right)=E_{r, s}\left(x_{1}, y_{1}\right)$.

Remark 2. We assume that there exists $i, 1 \leq i \leq n$, such that $x_{i} \neq y_{i}$.

Proof. Properties 2-6 are self-evident. For the rest of the proof we can assume that $x_{i}>y_{i}, i=1,2, \ldots, n$. Otherwise, we put $x_{i} \in Y_{n}, y_{i} \in X_{n}$.

Furthermore, because of symmetry, we take $s \geq r$.

To prove Property 1 , note that from the definition of Stolarsky means, for $s>r \neq 0$ and each $i=1,2, \ldots, n$, the bounds

$$
y_{i} \leq\left(\frac{r\left(x_{i}^{s}-y_{i}^{s}\right)}{s\left(x_{i}^{r}-y_{i}^{r}\right)}\right)^{1 /(s-r)} \leq x_{i}
$$

are known.

Hence,

$$
\left(s\left(x_{i}^{r}-y_{i}^{r}\right)\right)^{2} y_{i}^{2(s-r)} \leq\left(r\left(x_{i}^{s}-y_{i}^{s}\right)\right)^{2} \leq\left(s\left(x_{i}^{r}-y_{i}^{r}\right)\right)^{2} x_{i}^{2(s-r)},
$$

and

$$
s^{2} \sum_{i=1}^{n} a_{i}\left(x_{i}^{r}-y_{i}^{r}\right)^{2} y_{i}^{2(s-r)} \leq r^{2} \sum_{i=1}^{n} a_{i}\left(x_{i}^{s}-y_{i}^{s}\right)^{2} \leq s^{2} \sum_{i=1}^{n} a_{i}\left(x_{i}^{r}-y_{i}^{r}\right)^{2} x_{i}^{2(s-r)},
$$

wherefrom one easily obtains that

$$
s^{2}\left(\min \left\{y_{i}\right\}\right)^{2(s-r)} \sum_{i=1}^{n} a_{i}\left(x_{i}^{r}-y_{i}^{r}\right)^{2} \leq r^{2} \sum_{i=1}^{n} a_{i}\left(x_{i}^{s}-y_{i}^{s}\right)^{2} \leq s^{2}\left(\max \left\{x_{i}\right\}\right)^{2(s-r)} \sum_{i=1}^{n} a_{i}\left(x_{i}^{r}-y_{i}^{r}\right)^{2},
$$

i.e.,

$$
\min \left\{y_{i}\right\} \leq\left(\frac{r^{2}\left(\sum_{1}^{n} a_{i}\left(x_{i}^{s}-y_{i}^{s}\right)^{2}\right.}{s^{2}\left(\sum_{1}^{n} a_{i}\left(x_{i}^{r}-y_{i}^{r}\right)^{2}\right.}\right)^{1 / 2(s-r)} \leq \max \left\{x_{i}\right\}, \quad i=1,2, \ldots, n .
$$

The other cases follow simultaneously as a results of limit processes inside the definite fixed bounds.

For example, for $r=s=0$, we have

$$
\begin{gathered}
E_{0,0}\left(A_{n} ; X_{n}, Y_{n}\right)=\exp \left(\frac{\sum_{1}^{n} a_{i}\left(\log x_{i}-\log y_{i}\right)\left(\log ^{2} x_{i}-\log ^{2} y_{i}\right)}{2 \sum_{1}^{n} a_{i}\left(\log x_{i}-\log y_{i}\right)^{2}}\right) \\
=\exp \left(\frac{\sum_{1}^{n} a_{i}\left(\log x_{i}-\log y_{i}\right)^{2} \log \left(\sqrt{x_{i} y_{i}}\right)}{\sum_{1}^{n} a_{i}\left(\log x_{i}-\log y_{i}\right)^{2}}\right),
\end{gathered}
$$

and applying the inequality

$$
\min \left\{y_{i}\right\} \leq y_{i} \leq \sqrt{x_{i} y_{i}} \leq x_{i} \leq \max \left\{x_{i}\right\}
$$

we obtain

$$
\min \left\{y_{i}\right\} \leq E_{0,0}\left(A_{n} ; X_{n}, Y_{n}\right) \leq \max \left\{x_{i}\right\}, \quad i=1,2, \ldots, n
$$


Therefore, the fact that expressions $E_{r, s}\left(A_{n} ; X_{n}, Y_{n}\right)$ are means is proved.

For the proof of Property 5., let us recall some basic facts from Convexity Theory.

A function $f$ is convex on an interval $I$ if it is continuous on $I$ and it is Jensen convex on $I$, that is for all $x, y \in I$,

$$
\frac{f(x)+f(y)}{2} \geq f\left(\frac{x+y}{2}\right) .
$$

Lemma 2. A positive function $g$ is log-convex on an interval I if it is continuous on I and the inequality

$$
\alpha^{2} g(s)+2 \alpha \beta g\left(\frac{s+t}{2}\right)+\beta^{2} g(t) \geq 0,
$$

holds for all $\alpha, \beta \in \mathbb{R}$ and $s, t \in I$.

Proof. The above inequality holds for all $\alpha, \beta \in \mathbb{R}$ if and only if

$$
g(s) g(t) \geq g^{2}\left(\frac{s+t}{2}\right)
$$

that is

$$
\frac{\log g(s)+\log g(t)}{2} \geq \log g\left(\frac{s+t}{2}\right) .
$$

This means that $\log \circ g$ is convex in the Jensen sense, and hence the continuity of $g$ implies that it is log-convex.

Lemma 3. Let the function $h(x, y ; s), x>y>0$, be defined as

$$
h(s)=h(x, y ; s):= \begin{cases}\frac{x^{s}-y^{s}}{s} & , s \neq 0 \\ \log (x / y) & , s=0\end{cases}
$$

Then $h(s)$ is log-convex on $s \in \mathbb{R}$.

Proof. Indeed, $h(s)$ is continuous on $s \in \mathbb{R}$ and the inequality

$$
\alpha^{2} h(s)+2 \alpha \beta h\left(\frac{s+t}{2}\right)+\beta^{2} h(t) \geq 0
$$

holds, because

$$
\begin{gathered}
\alpha^{2} h(s)+2 \alpha \beta h\left(\frac{s+t}{2}\right)+\beta^{2} h(t) \\
=\alpha^{2} \int_{y}^{x} u^{s-1} d u+2 \alpha \beta \int_{y}^{x} u^{\frac{s+t}{2}-1} d u+\beta^{2} \int_{y}^{x} u^{t-1} d u \\
=\int_{y}^{x}\left(\alpha u^{s / 2}+\beta u^{t / 2}\right)^{2} u^{-1} d u .
\end{gathered}
$$

Therefore Lemma 2 can be applied.

Lemma 4. If, for positive $u, v, w$, the inequality

$$
\alpha^{2} u+2 \alpha \beta v+\beta^{2} w \geq 0
$$

holds for each $\alpha, \beta \in \mathbb{R}$, then also

$$
\alpha^{2} u^{p}+2 \alpha \beta v^{p}+\beta^{2} w^{p} \geq 0
$$


holds for each $p>0$.

Proof. Obvious. Now we are enabled to prove Property 5. of Theorem 4. For this cause, denote

$$
g_{n}(s)=\sum_{1}^{n} a_{i} h_{i}^{2}(s)
$$

where $h_{i}(s):=h\left(x_{i}, y_{i} ; s\right)$ and $a_{i}, i=1,2, \ldots, n$ are positive numbers.

By Lemmas $2-4$, we see that $g_{n}(s)$ is log-convex in $s, s \in \mathbb{R}$, since

$$
\alpha^{2} g_{n}(s)+2 \alpha \beta g_{n}\left(\frac{s+t}{2}\right)+\beta^{2} g_{n}(t)=\sum_{1}^{n} a_{i}\left(\alpha^{2} h_{i}^{2}(s)+2 \alpha \beta h_{i}^{2}\left(\frac{s+t}{2}\right)+\beta^{2} h_{i}^{2}(t)\right) \geq 0 .
$$

Therefore the function $F(s)=\log g_{n}(s)$ is convex and, applying Lemma 1, we obtain that

$$
\frac{\log g_{n}(s)-\log g_{n}(r)}{s-r}=\log \left(\frac{g_{n}(s)}{g_{n}(r)}\right)^{\frac{1}{s-r}}=2 \log E_{r, s}\left(A_{n} ; X_{n}, Y_{n}\right),
$$

is monotone increasing in both $r$ and $s$, which is equivalent with the Property 5 in the case $s>r \neq 0$.

By continuity, the proof of other cases follows immediately. For example, since for any $\epsilon>0$ we have

$$
E_{r+\epsilon, s+\epsilon}\left(A_{n} ; X_{n}, Y_{n}\right) \geq E_{r, s}\left(A_{n} ; X_{n}, Y_{n}\right)
$$

letting $r \rightarrow s$, we obtain

$$
E_{s+\epsilon, s+\epsilon}\left(A_{n} ; X_{n}, Y_{n}\right) \geq E_{s, s}\left(A_{n} ; X_{n}, Y_{n}\right),
$$

that is, $E_{s, s}\left(A_{n} ; X_{n}, Y_{n}\right)$ is monotone increasing in $s$.

Our task in the sequel is to investigate under what conditions the means $e_{r, s}\left(X_{n}\right)$ and $E_{r, s}\left(A_{n} ; X_{n}, Y_{n}\right)$ are monotone increasing/decreasing in $n$.

For this cause we need the following two lemmas.

Lemma 5. Stolarsky means $E_{r, s}(x, y)$ are monotone increasing in both variables $x$ and $y$.

This is the well-known assertion ([1]).

Lemma 6. For two given sequences $\left\{u_{n}\right\}$ and $\left\{v_{n}\right\}$ of positive numbers, denote

$$
w_{n}:=\frac{u_{n}}{v_{n}} ; W_{n}:=\frac{u_{1}+u_{2}+\ldots+u_{n}}{v_{1}+v_{2}+\ldots+v_{n}} .
$$

If the sequence $w_{n}$ is monotone decreasing/increasing, then the sequence $W_{n}$ is also monotone decreasing/increasing.

Proof. Let $w_{n}$ be a decreasing sequence. The other case can be treated similarly.

We prove firstly that $v_{n+1} \sum_{1}^{n} u_{i} \geq u_{n+1} \sum_{1}^{n} v_{i}$.

Indeed,

$$
v_{n+1} \sum_{1}^{n} u_{i}=v_{n+1} \sum_{1}^{n} v_{i} w_{i} \geq v_{n+1} w_{n+1} \sum_{1}^{n} v_{i}=u_{n+1} \sum_{1}^{n} v_{i}
$$

Hence, 


$$
\sum_{1}^{n} u_{i} \sum_{1}^{n+1} v_{i}=\sum_{1}^{n} u_{i} \sum_{1}^{n} v_{i}+v_{n+1} \sum_{1}^{n} u_{i} \geq \sum_{1}^{n} u_{i} \sum_{1}^{n} v_{i}+u_{n+1} \sum_{1}^{n} v_{i}=\sum_{1}^{n} v_{i} \sum_{1}^{n+1} u_{i},
$$

i.e., $W_{n} \geq W_{n+1}$.

Theorem 5. If both sequences $\left\{X_{n}\right\}$ and $\left\{Y_{n}\right\}$ are monotone decreasing (increasing), then means $E_{r, s}\left(A_{n} ; X_{n}, Y_{n}\right)$ are monotone decreasing (increasing) in $n$.

Proof. We shall prove the "decreasing" part of Theorem 5. The proof of the other part is analogous.

Hence, we assume that both sequences $\left\{X_{n}\right\}$ and $\left\{Y_{n}\right\}$ are monotone decreasing. In the case $s>r \neq 0$, denote

$$
u_{n}:=a_{n} r^{2}\left(x_{n}^{s}-y_{n}^{s}\right)^{2} ; v_{n}:=a_{n} s^{2}\left(x_{n}^{r}-y_{n}^{r}\right)^{2} .
$$

By Lemma 5, we have

$$
w_{n}=\frac{u_{n}}{v_{n}}=\frac{r^{2}\left(x_{n}^{s}-y_{n}^{s}\right)^{2}}{s^{2}\left(x_{n}^{r}-y_{n}^{r}\right)^{2}} \geq \frac{r^{2}\left(x_{n+1}^{s}-y_{n+1}^{s}\right)^{2}}{s^{2}\left(x_{n+1}^{r}-y_{n+1}^{r}\right)^{2}}=w_{n+1} .
$$
that is,

Therefore the sequence $w_{n}$ is monotone decreasing and, by Lemma 6, this implies $W_{n} \geq W_{n+1}$,

$$
\begin{gathered}
\frac{r^{2}}{s^{2}} \frac{a_{1}\left(x_{1}^{s}-y_{1}^{s}\right)^{2}+a_{2}\left(x_{2}^{s}-y_{2}^{s}\right)^{2}+\cdots+a_{n}\left(x_{n}^{s}-y_{n}^{s}\right)^{2}}{a_{1}\left(x_{1}^{r}-y_{1}^{r}\right)^{2}+a_{2}\left(x_{2}^{r}-y_{2}^{r}\right)^{2}+\cdots+a_{n}\left(x_{n}^{r}-y_{n}^{r}\right)^{2}} \\
\geq \frac{r^{2}}{s^{2}} \frac{a_{1}\left(x_{1}^{s}-y_{1}^{s}\right)^{2}+a_{2}\left(x_{2}^{s}-y_{2}^{s}\right)^{2}+\cdots+a_{n}\left(x_{n}^{s}-y_{n}^{s}\right)^{2}+a_{n+1}\left(x_{n+1}^{s}-y_{n+1}^{s}\right)^{2}}{a_{1}\left(x_{1}^{r}-y_{1}^{r}\right)^{2}+a_{2}\left(x_{2}^{r}-y_{2}^{r}\right)^{2}+\cdots+a_{n}\left(x_{n}^{r}-y_{n}^{r}\right)^{2}+a_{n+1}\left(x_{n+1}^{r}-y_{n+1}^{r}\right)^{2}} .
\end{gathered}
$$

Since $s>r$, this is equivalent to $E_{r, s}\left(A_{n} ; X_{n}, Y_{n}\right) \geq E_{r, s}\left(A_{n+1} ; X_{n+1}, Y_{n+1}\right)$.

In the cases $r=s \neq 0, s>0=r$ and $r=s=0$ one should take

$$
\begin{gathered}
u_{n}=a_{n}\left(x_{n}^{s}-y_{n}^{s}\right)\left(x_{n}^{s} \log x_{i}-y_{n}^{s} \log y_{i}\right), v_{n}=a_{n}\left(x_{n}^{s}-y_{n}^{s}\right)^{2} ; \\
u_{n}=a_{n}\left(x_{n}^{s}-y_{n}^{s}\right)^{2}, v_{n}=s^{2} a_{n}\left(\log x_{n}-\log y_{n}\right)^{2} ; \\
u_{n}=a_{n}\left(\log x_{n}-\log y_{n}\right)\left(\log ^{2} x_{n}-\log ^{2} y_{n}\right), v_{n}=2 a_{n}\left(\log x_{n}-\log y_{n}\right)^{2},
\end{gathered}
$$

respectively, and proceed as above.

On the other hand, the problem of monotonicity in $n$ for means $e_{r, s}\left(X_{n}\right)$ seems significantly harder. We are able to solve it only in the simplest case $r=s=0$.

Theorem 6. The means $e_{0,0}\left(X_{n}\right)$ are monotone increasing/decreasing in $n$ if and only if

$$
x_{n} \gtrless z\left(X_{n-1}\right)\left(e_{0,0}\left(X_{n-1}\right) / z\left(X_{n-1}\right)\right)^{3 n /(n+1)}, n \geq 3,
$$

where $z\left(X_{n}\right)$ denotes the geometric mean of numbers $X_{n}$.

Proof. We have

$$
\log e_{0,0}\left(X_{n}\right)=\frac{b_{n}\left(X_{n}\right)}{3 a_{n}\left(X_{n}\right)}
$$

with 


$$
a_{n}\left(X_{n}\right)=\frac{1}{n} \sum_{1}^{n} \log ^{2} x_{i}-\left(\frac{1}{n} \sum_{1}^{n} \log x_{i}\right)^{2}=\frac{1}{n} \sum_{1}^{n} \log ^{2}\left(x_{i} / z\left(X_{n}\right)\right) \geq 0
$$

and

$$
b_{n}\left(X_{n}\right)=\frac{1}{n} \sum_{1}^{n} \log ^{3} x_{i}-\left(\frac{1}{n} \sum_{1}^{n} \log x_{i}\right)^{3} .
$$

Note that for $x_{n}=z\left(X_{n-1}\right)$ we have $e_{0,0}\left(X_{n}\right)=e_{0,0}\left(X_{n-1}\right)$. Therefore by Taylor expansion around this point, we obtain

$$
a_{n}\left(X_{n}\right)=\frac{n-1}{n}\left[a_{n-1}\left(X_{n-1}\right)+\frac{1}{n} \log ^{2}\left(x_{n} / z\left(X_{n-1}\right)\right)\right],
$$

and

$$
b_{n}\left(X_{n}\right)=\frac{n-1}{n}\left[b_{n-1}\left(X_{n-1}\right)+\frac{3 \log z\left(X_{n-1}\right)}{n} \log ^{2}\left(x_{n} / z\left(X_{n-1}\right)\right)+\frac{n+1}{n^{2}} \log ^{3}\left(x_{n} / z\left(X_{n-1}\right)\right)\right] .
$$

Since $b_{n-1}\left(X_{n-1}\right) / 3 a_{n-1}\left(X_{n-1}\right)=\log e_{0,0}\left(X_{n-1}\right)$, we finally get

$$
\begin{gathered}
\log e_{0,0}\left(X_{n}\right)-\log e_{0,0}\left(X_{n-1}\right)=\frac{b_{n}\left(X_{n}\right)}{3 a_{n}\left(X_{n}\right)}-\frac{b_{n-1}\left(X_{n-1}\right)}{3 a_{n-1}\left(X_{n-1}\right)} \\
=\frac{\log ^{2}\left(x_{n} / z\left(X_{n-1}\right)\right)}{3 n a_{n}\left(X_{n}\right)}\left[3 \log z\left(X_{n-1}\right)+\frac{n+1}{n} \log \left(x_{n} / z\left(X_{n-1}\right)\right)-b_{n-1}\left(X_{n-1}\right) / a_{n-1}\left(X_{n-1}\right)\right],
\end{gathered}
$$

and the proof follows.

\section{Conclusions}

In this article we give two explicit generalizations of Stolarsky means to the multi-variable case and proved that they preserve all main properties of the original means. Let us note that other subtle properties are not equally transposed. For example, log-convexity of $E_{r, s}\left(x_{1}, x_{2}\right)$ entirely depends on parameters $r, s$ ([12]), but in the case of means $B_{r, s}\left(x_{1}, x_{2}, x_{3}\right)$, mentioned in the Introduction, it also depends on $x_{3} \lessgtr \sqrt{x_{1} x_{2}}$.

Furthermore, many open questions can be proposed. For example, is monotone increase of the sequences $\left\{X_{n}\right\}$ and $\left\{Y_{n}\right\}$ necessary for $E_{r, s}\left(A_{n} ; X_{n}, Y_{n}\right)$ to be increasing in $n$ ?

Or, is the monotonicity in variables possible for the means $e_{r, s}\left(X_{n}\right)$ only if $n=2$ or $n=3$ ?

Author Contributions: Theoretical part, S.S.; numerical part with examples, B.B.-M. All authors have read and agreed to the published version of the manuscript.

Funding: Bandar Bin-Mohsin is supported by Researchers Supporting Project number (RSP-2020/158), King Saud University, Riyadh, Saudi Arabia.

Acknowledgments: The authors are grateful to the referees for their valuable comments.

Conflicts of Interest: The authors declare no conflict of interests.

\section{References}

1. Stolarsky, K.B. Generalizations of the logarithmic mean. Math. Mag. 1995, 48, 87-92. [CrossRef]

2. Simic, S. Another converse of Jensen's Inequality. Available online: http://vuir.vu.edu.au/17742/1/simic. pdf (accessed on 20 March 2020).

3. Stolarsky, K.B. The power and generalized logarithmic means. Amer. Math. Mon. 1980, 87, 545-548. [CrossRef]

4. Leach E.B.; Sholander, M.C. Extended mean values. Amer. Math. Mon. 1978, 85, 84-90. [CrossRef] 
5. Leach, E.B.; Sholander, M.C. Multi-variable extended mean values. J. Math. Anal. Appl. 1984, 104, $390-407$. [CrossRef]

6. Páles, Z. Inequalities for differences of powers. J. Math. Anal. Appl. 1988, 131, 271-281. [CrossRef]

7. Neuman E.; Páles, Z. On comparison of Stolarsky and Gini means. J. Math. Anal. Appl. 2003, 278, $274-284$. [CrossRef]

8. Neuman E.; Sándor, J. Inequalities involving Stolarsky and Gini means. Math. Pannonica 2003, 14, $29-44$.

9. Czinder, P.; Páles, Z. An extension of the Hermite-Hadamard inequality and an application for Gini and Stolarsky means. J. Ineq. Pure Appl. Math. 2004, 5, 42.

10. Witkowski, A. Weighted extended mean values. Colloq. Math. 2004, 100, 111-117. [CrossRef]

11. Witkowski, A. Convexity of weighted Stolarsky means. J. Inequal. Pure Appl. Math. 2006, 7, 73.

12. Qi, F. Logarithmic convexity of extended mean values. Proc. Amer. Math. Soc. 2001, 130, 1787-1796. [CrossRef]

13. Merikoski, J. K. Extending means of two variables to several variables. J. Ineq. Pure Appl. Math. 2004, 5, 65.

14. Pečarić, J.; Šimić, V. Stolarsky-Tobey mean in $n$ variables. Math. Ineq. Appl. 1999, 2, 325-334. [CrossRef]

15. Horvath, I.; Pečarić, J. New versions of weighted multidimensional functional and Stolarsky means. Acta Math. Hung. 2015, 147, 81-96. [CrossRef]

16. Simic, S. On a class of mean values including Jensen functionals. Publ. Math. Debrecen 2008, 73, 461-469.

17. Simic, S. An extension of Stolarsky means to the multivariable case. Int. J. Math. Math. Sci. 2009, 32857. [CrossRef]

18. Simic, S. On certain new inequalities in Information Theory. Acta Math. Hungar. 2009, 124, $353-361$. [CrossRef]

(C) 2020 by the authors. Licensee MDPI, Basel, Switzerland. This article is an open access article distributed under the terms and conditions of the Creative Commons Attribution (CC BY) license (http:/ / creativecommons.org/licenses/by/4.0/). 